\title{
The sum of the largest and smallest signless laplacian eigenvalues and some Hamiltonian properties of graphs
}

\author{
Rao Li ${ }^{1 *}$
}

\begin{abstract}
The signless Laplacian eigenvalues of a graph $G$ are eigenvalues of the matrix $Q(G)=D(G)+A(G)$, where $D(G)$ is the diagonal matrix of the degrees of the vertices in $G$ and $A(G)$ is the adjacency matrix of $G$. Using a result on the sum of the largest and smallest signless Laplacian eigenvalues obtained by Das in [2], we in this note present sufficient conditions based on the sum of the largest and smallest signless Laplacian eigenvalues for some Hamiltonian properties of graphs.
\end{abstract}

Keywords: Signless Laplacian Eigenvalues, Hamiltonian Properties

2010 AMS: Primary 05C50, Secondary 05C45

${ }^{1}$ Department of Mathematical Sciences, University of South Carolina Aiken, Aiken, SC 29801, USA

*Corresponding author: raol@usca.edu

Received: 13 July 2018, Accepted: 8 September 2018, Available online: 30 September 2018

\section{Introduction}

We consider only finite undirected graphs without loops or multiple edges. Notation and terminology not defined here follow those in [1]. For a graph $G=(V(G), E(G))$, we use $n$ to denote its order $|V(G)|$. A subset $V_{1}$ of the vertex set $V(G)$ is independent if no two vertices in $V_{1}$ are adjacent in $G$. The size of a maximum independent set is called the independence number of $G$ and it is denoted by $\alpha(G)$. We use $G_{1} \vee G_{2}$ to denote the the join of two disjoint graphs $G_{1}$ and $G_{2}$. The graph consists of $p$ isolated vertices is denoted by $p K_{1}$. Let $D(G)$ be a diagonal matrix such that its diagonal entries are the degrees of vertices in a graph $G$. The signless Laplacian matrix of a graph $G$, denoted $Q(G)$, is defined as $D(G)+A(G)$, where $A(G)$ is the adjacency matrix of $G$. The eigenvalues $q_{1}(G) \geq q_{2}(G) \geq \cdots \geq q_{n}(G)$ of $Q(G)$ are called the signless Laplacian eigenvalues of $G$. A cycle $C$ in a graph $G$ is called a Hamiltonian cycle of $G$ if $C$ contains all the vertices of $G$. A graph $G$ is called Hamiltonian if $G$ has a Hamiltonian cycle. A path $P$ in a graph $G$ is called a Hamiltonian path of $G$ if $P$ contains all the vertices of $G$. A graph $G$ is called traceable if $G$ has a Hamiltonian path.

In this note we present sufficient conditions based on the sum of the largest and smallest signless Laplacian eigenvalues for the Hamiltonian and traceable graphs. The main results are as follows.

Theorem 1.1. Let $G$ be a $k$-connected graph $(k \geq 2)$ of order $n \geq 4$. If $q_{1}+q_{n} \geq 3 n-2 k-4$, then $G$ is Hamiltonian or $G$ is $(k+1) K_{1} \vee K_{r}$ with $2 \leq r \leq k$. 
Theorem 1.2. Let $G$ be a $k$-connected $(k \geq 1)$ graph of order $n \geq 4$. If $q_{1}+q_{n} \geq 3 n-2 k-6$, then $G$ is traceable or $G$ is $(k+2) K_{1} \vee K_{r}$ with $1 \leq r \leq k$.

\section{Proofs}

In order to prove Theorem 1.1 and Theorem 1.2, we need the following result obtained by Das as our lemma. Lemma 2.1 below is Theorem 3.2 on Page 995 in [2].

Lemma 2.1. Let $G$ be a connected graph on $n \geq 4$ vertices with independence number $\alpha$. Then $q_{1}+q_{n}+2 \alpha \leq 3 n-2$ with equality holding if and only if $G$ is $\alpha K_{1} \vee K_{n-\alpha}$.

Proof of Theorem 1. Let $G$ be a graph satisfying the conditions in Theorem 1.1. Suppose, to the contrary, that $G$ is not Hamiltonian. Since $k \geq 2, G$ has a cycle. Choose a longest cycle $C$ in $G$ and give an orientation on $C$. Since $G$ is not Hamiltonian, there exists a vertex $u_{0} \in V(G)-V(C)$. By Menger's theorem, we can find $k$ pairwise disjoint (except for $u_{0}$ ) paths $P_{1}, P_{2}, \ldots, P_{k}$ between $u_{0}$ and $V(C)$. Let $v_{i}$ be the end vertex of $P_{i}$ on $C$, where $1 \leq i \leq k$. Without loss of generality, we assume that the appearance of $v_{1}, v_{2}, \ldots, v_{k}$ agrees with the orientation of $C$. We use $v_{i}^{+}$to denote the successor of $v_{i}$ along the orientation of $C$, where $1 \leq i \leq k$. Since $C$ is a longest cycle in $G$, we have that $v_{i}^{+} \neq v_{i+1}$, where $1 \leq i \leq k$ and the index $k+1$ is regarded as 1 . Moreover, $S:=\left\{u_{0}, v_{1}^{+}, v_{2}^{+}, \ldots, v_{k}^{+}\right\}$is independent (otherwise $G$ would have cycles which are longer than $C$ ). From Lemma 2.1, we have that

$$
3 n-2=3 n-2 k-4+2(k+1) \leq q_{1}+q_{n}+2|S| \leq q_{1}+q_{n}+2 \alpha \leq 3 n-2 .
$$

From Lemma 2.1 again, we have that $q_{1}+q_{n}=3 n-2 k-4, S$ is a maximum independent set of size $\alpha=k+1$, and $G$ is $(k+1) K_{1} \vee K_{n-(k+1)}$. Notice that $G$ is Hamiltonian if $n-(k+1) \geq(k+1)$. Thus $n-(k+1) \leq k$. Since $G$ is $k$-connected with $k \geq 2, G$ must be $(k+1) K_{1} \vee K_{r}$ with $2 \leq r \leq k$.

Proof of Theorem 2. Let $G$ be a graph satisfying the conditions in Theorem 1.2. Suppose, to the contrary, that $G$ is not traceable. Choose a longest path $P$ in $G$ and give an orientation on $P$. Let $x$ and $y$ be the two end vertices of $P$. Since $G$ is not traceable, there exists a vertex $u_{0} \in V(G)-V(P)$. By Menger's theorem, we can find $k$ pairwise disjoint (except for $u_{0}$ ) paths $P_{1}, P_{2}, \ldots, P_{k}$ between $u_{0}$ and $V(P)$. Let $v_{i}$ be the end vertex of $P_{i}$ on $P$, where $1 \leq i \leq s$. Without loss of generality, we assume that the appearance of $v_{1}, v_{2}, \ldots, v_{k}$ agrees with the orientation of $P$. Since $P$ is a longest path in $G, x \neq v_{i}$ and $y \neq v_{i}$, for each $i$ with $1 \leq i \leq k$, otherwise $G$ would have paths which are longer than $P$. We use $v_{i}^{+}$to denote the successor of $v_{i}$ along the orientation of $P$, where $1 \leq i \leq k$. Since $P$ is a longest path in $G$, we have that $v_{i}^{+} \neq v_{i+1}$, where $1 \leq i \leq k-1$. Moreover, $\left\{u_{0}, v_{1}^{+}, v_{2}^{+}, \ldots, v_{k}^{+}, x\right\}$ is independent (otherwise $G$ would have paths which are longer than $P$ ). From Lemma 2.1, we have that

$$
3 n-2=3 n-2 k-6+2(k+2) \leq q_{1}+q_{n}+2|S| \leq q_{1}+q_{n}+2 \alpha \leq 3 n-2 .
$$

From Lemma 2.1 again, we have that $q_{1}+q_{n}=3 n-2 k-6, S$ is a maximum independent set of size $\alpha=k+2$, and $G$ is $(k+2) K_{1} \vee K_{n-(k+2)}$. Notice that $G$ is traceable if $n-(k+2) \geq(k+1)$. Thus $n-(k+2) \leq k$. Since $G$ is $k$-connected with $k \geq 1, G$ must be $(k+2) K_{1} \vee K_{r}$ with $1 \leq r \leq k$.

\section{References}

[1] J. A. Bondy and U. S. R. Murty, Graph Theory with Applications, Macmillan, London and Elsevier, New York (1976).

[2] K. C. Das, Proof of conjectures involving the largest and the smallest signless Laplacian eigenvalues of graphs, Discrete Mathematics 312 (2012) 992 - 998. 